\title{
Theoretical analysis, numerical calculation and experimental measurement of transient elastic waves in strips subjected to dynamic loadings

\author{
Chien-Ching Ma*, Genn-Sheng Lee \\ Department of Mechanical Engineering, National Taiwan University, Taipei, Taiwan 10617, People's Republic of China
}

Received 14 January 1998; in revised form 28 April 1998

\begin{abstract}
In this study, the transient response of an elastic strip subjected to dynamic in-plane loadings on the surface is investigated in detail. One of the objectives of this study is to develop an effective analytical method for determining transient solutions in a strip. By applying Laplace transform, the analytical solution in the transformed domain is derived and expressed in matrix form. The solution is then decomposed into infinite wave groups in which the multiple reflected waves with the same reflection are involved. Each multireflected wave can be identified by a coding method and be verified by the theory of generalized ray. The inverse transform is performed by using the well-known Cagniard method. The transient solutions in time domain for stresses and displacements are expressed in a closed form and are discussed in detail by an example. The experimental results show that the early time transient responses of displacements on the surface agree very well with the numerical calculations based on the theoretical solutions. (C) 1999 Elsevier Science Ltd. All rights reserved.
\end{abstract}

\section{Introduction}

The dynamic transient responses for a plate subjected to dynamic loading are of great interest in many engineering applications. Analysis of transient elastic waves generated by dynamic loading in a plate has been investigated widely in the literature. The flexural waves and extensional waves in a thin plate were analyzed by the classical plate theory (Miklowitz, 1960, 1962). Weaver and Pao (1982) calculated the transient response of an axisymmetric point source acting on a thick plate by using the theory of normal modes. Santosa and Pao (1989) calculated the asymmetric one. The normal mode method is effective in determining the long time response at a receiver which is located a long distance from the source, but the method is inefficient in determining the

* Corresponding author. Fax: 00886223631755

0020-7683/99/\$ - see front matter (C) 1999 Elsevier Science Ltd. All rights reserved PII: S $0020-7683(98) 00155-3$ 
early time response at a receiver relatively close to the source. The early time response in the plate can be determined exactly by considering the propagation of stress waves.

The propagation of stress waves through an unbounded medium is not a difficult subject. If a boundary is introduced, however, reflected waves will be generated from the free surface, making the problem more complicated. The classical analysis in this area was first proposed by Lamb (1904); he considered a half-space subjected to point and line loads on the surface. Since this early analysis of Lamb, a great many contributions have appeared, pertaining to what is commonly referred to as Lamb's problem. De Hoop (1961) and Cagniard (1939) proposed a powerful and elegantly simple method that is known as the Cagniard-de Hoop technique for inverting transforms in a wide range of elastodynamic wave propagation problems. Spancer (1960) presented a method to predict the response of an elastic system that consists of two half spaces perfectly bounded together along a plane interface. He showed that the integral representation of reflected and transmission waves can be obtained from source waves by suitable operation. This concept is the foundation of the generalized ray. The theory of generalized ray was reviewed by Pao and Gajewski (1979). To analyze the transient response of a plate, the solution was decomposed into wave components called 'rays'. Each ray can be evaluated exactly by Cagniard's method. Although the number of waves in the plate is infinite, the solution is exact up to the arrival time of the next ray. The ray solution is suitable for calculating the early time response. The numerical results of various types of point source in a plate analyzed by the generalized ray method were given by Ceranoglu and Pao (1981).

Various modified methods were presented to obtain the transient solutions of a plate. Mencher (1953) expanded the solution of a symmetric plate problem into a series of exponential functions and each term representing a generalized ray. Knopoff (1958) and Davids (1959) used a similar expanding procedure to obtain the ray solutions for both symmetric and antisymmetric plate problems. They expanded the denominator in the expression of solution into a series of exponential functions formally but only the first few terms were considered. On the other hand, Shmuely (1974) and Norwood (1975) derived a solution by a ray tracing method which was greatly simplified by matrix notation. Whenever these waves reach any one of the two surfaces, a half-space sort of solution is added to the solution. The final solution thus consists of infinitely many contributions, each of which corresponds to a reflection from one of the surfaces.

Because of the difficulty in analyzing the transient response of an infinite number of reflected waves in a strip, only very few papers used the transient analysis to study the phenomena of the wave propagation in a strip. For transient waves, one could in principle obtain the solution by superimposing harmonic waves of all frequencies and all modes. This approach, however, is not practical and a more direct approach should be employed to study the transient waves. In this study, a matrix expansion method is proposed and will be demonstrated as an efficient methodology to solve the problem. By applying the Laplace transform, the stress vector in the strip is expressed in terms of a matrix form in transform domain. From the boundary conditions at lateral surfaces, a system of equations with a coefficient matrix is derived. By rewriting the coefficient matrix in a special form which consists of the diagonal, lower and upper triangular parts and then expanding the inversion of coefficient matrix into a power matrix series, the solution in transform domain can be automatically decomposed into finite wave groups in which the multiple reflected waves with the same reflections are involved. We found that the source function should be separated before expanding the denominator into a series of rays. The physical meaning of the matrix 
representation will be clear under this arrangement. The connection of the matrix formulation and generalized rays is also discussed in this study. The solution can be expressed in a summation form by encoding each individual ray and then each ray can be easily traced by the coding. The inverse Laplace transform is achieved by Cagniard's method. All the waves are summed to construct the complete transient solution in time domain. Numerical examples are given when the applied loading is a point vertical force with a step time dependence. Experimental data of transient responses for displacements on lateral surfaces are compared with the theoretical predictions, and good agreement is obtained.

\section{Statements of the problem}

Consider an infinite strip with thickness $h$ subjected to arbitrarily distributed dynamic forces applied on the top and lower surfaces. A Cartesian coordinate system is oriented so that the $y$-axis is normal to the surfaces as shown in Fig. 1. Only in-plane motion in the $x-y$ plane is considered in this study. The top surface lies in the plane $y=0$ and the lower one in $y=-h$. The boundary conditions on the top and lower surfaces of the problem can be written as follows

$$
\begin{aligned}
& \left\{\begin{array}{l}
\sigma_{x y}(x, 0, t)=\sigma_{x y_{0}}(x, t) \\
\sigma_{y y}(x, 0, t)=\sigma_{y y_{0}}(x, t)
\end{array}-\infty<x<\infty,\right. \\
& \left\{\begin{array}{l}
\sigma_{x y}(x,-h, t)=\sigma_{x y_{h}}(x, t) \\
\sigma_{y y}(x,-h, t)=\sigma_{y y_{h}}(x, t)
\end{array}-\infty<x<\infty,\right.
\end{aligned}
$$

where $\sigma_{x y_{0}}(x, t), \ldots, \sigma_{y y_{h}}(x, t)$ are applied tractions on the surfaces.

The two-dimensional elastodynamic equations without body forces can be expressed in terms of two scalar potentials $\phi$ and $\psi$ as follows

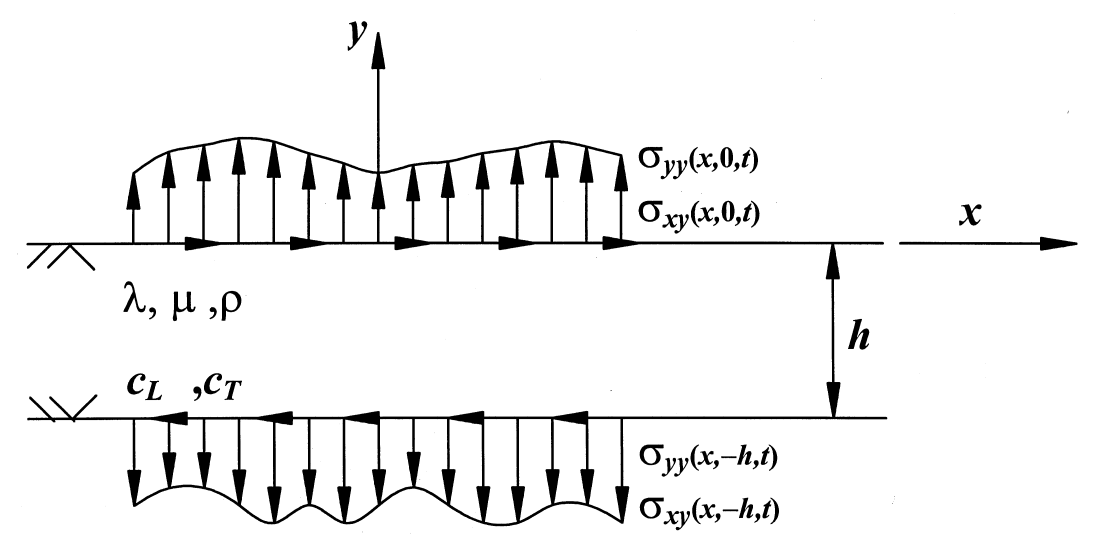

Fig. 1. Configuration and coordinate systems of a strip. 


$$
\begin{aligned}
& \frac{\partial^{2} \phi}{\partial x^{2}}+\frac{\partial^{2} \phi}{\partial y^{2}}=s_{\mathrm{L}}^{2} \frac{\partial^{2} \phi}{\partial t^{2}}, \\
& \frac{\partial^{2} \psi}{\partial x^{2}}+\frac{\partial^{2} \psi}{\partial y^{2}}=s_{\mathrm{T}}^{2} \frac{\partial^{2} \psi}{\partial t^{2}},
\end{aligned}
$$

where

$$
s_{\mathrm{L}}=\sqrt{\frac{\rho}{\lambda+2 \mu}}=\frac{1}{c_{\mathrm{L}}}, \quad s_{\mathrm{T}}=\sqrt{\frac{\rho}{\mu}}=\frac{1}{c_{\mathrm{T}}} .
$$

Here $\rho$ is the mass density of the material, $\lambda$ and $\mu$ are Lamé's constants, $s_{\mathrm{L}}$ and $s_{\mathrm{T}}$ are the slownesses of longitudinal and shear waves, respectively. The $\phi$ and $\psi$ are referred to as the p- and s-wave potentials. Displacements are derived from these potentials according to

$$
\begin{aligned}
& u=\frac{\partial \phi}{\partial x}+\frac{\partial \psi}{\partial y}, \\
& v=\frac{\partial \phi}{\partial y}-\frac{\partial \psi}{\partial x},
\end{aligned}
$$

where $u$ and $v$ are the displacements in the $x$ - and $y$-directions, respectively, The stresses can be expressed in terms of the two potentials by means of Hooke's law. The relevant components of the stress tensor can be written as

$$
\begin{aligned}
& \sigma_{x x}=\lambda\left(\frac{\partial^{2} \phi}{\partial x^{2}}+\frac{\partial^{2} \phi}{\partial y^{2}}\right)+2 \mu\left(\frac{\partial^{2} \phi}{\partial x^{2}}+\frac{\partial^{2} \psi}{\partial x \partial y}\right), \\
& \sigma_{y y}=\lambda\left(\frac{\partial^{2} \phi}{\partial x^{2}}+\frac{\partial^{2} \phi}{\partial y^{2}}\right)+2 \mu\left(\frac{\partial^{2} \phi}{\partial y^{2}}-\frac{\partial^{2} \psi}{\partial x \partial y}\right), \\
& \sigma_{x y}=\mu\left(2 \frac{\partial^{2} \phi}{\partial x \partial y}+\frac{\partial^{2} \psi}{\partial y^{2}}-\frac{\partial^{2} \psi}{\partial x^{2}}\right) .
\end{aligned}
$$

The aforementioned problem will be solved by the application of integral transformation. The one-sided Laplace transform over time $t$ and the bilateral Laplace transform on the spatial variable $x$ for a function $\phi$ is defined as

$$
\bar{\phi}^{*}(y ; \eta, p)=\int_{-\infty}^{\infty} \mathrm{e}^{-p \eta x} \int_{-0}^{\infty} \phi(x, y, t) \mathrm{e}^{-p t} \mathrm{~d} t \mathrm{~d} x,
$$

where $p$ is a positive real number, large enough to ensure the convergence of the integral and $\eta$ being a complex variable. For applying Cagniard's method of Laplace inversion, it is convenient to take $p \eta$ as the transform parameter of $x$.

The equations of motion in the Laplace transform domain are two ordinary differential equations with the following general solutions: 


$$
\begin{aligned}
& \bar{\phi}^{*}(y ; p, \eta)=\bar{\phi}_{\mathrm{d}}^{*}(p, \eta) \mathrm{e}^{p \gamma_{\mathrm{L}} y}+\bar{\phi}_{\mathrm{u}}^{*}(p, \eta) \mathrm{e}^{-p \gamma_{\mathrm{L}} y}, \\
& \bar{\psi}^{*}(y ; p, \eta)=\bar{\psi}_{\mathrm{d}}^{*}(p, \eta) \mathrm{e}^{p \gamma_{\mathrm{T}} y}+\bar{\psi}_{\mathrm{u}}^{*}(p, \eta) \mathrm{e}^{-p \gamma_{\mathrm{T}} y}
\end{aligned}
$$

where $\gamma_{\mathrm{L}}=\sqrt{s_{\mathrm{L}}^{2}-\eta^{2}}$ and $\gamma_{\mathrm{T}}=\sqrt{s_{\mathrm{T}}^{2}-\eta^{2}}$. The condition $\operatorname{Re} \gamma_{\mathrm{L}} \geqslant 0\left(\operatorname{Re} \gamma_{\mathrm{T}} \geqslant 0\right)$ is satisfied by providing branch cut along $s_{\mathrm{L}} \leqslant|\operatorname{Re} \eta| \leqslant \infty\left(s_{\mathrm{T}} \leqslant|\operatorname{Re} \eta| \leqslant \infty\right)$, $\operatorname{Im} \eta=0$ and choosing the branch of positive square root. The coefficients expressed in (5a) and (5b) with subscript $u$ denote the waves propagating along $+y$ direction and those with subscript $d$ are the waves propagating along $-y$ direction. The unknown coefficients $\bar{\phi}_{\mathrm{u}}^{*}, \bar{\psi}_{\mathrm{u}}^{*}, \bar{\phi}_{\mathrm{d}}^{*}$, and $\bar{\psi}_{\mathrm{d}}^{*}$, can be determined by boundary conditions.

The displacement and stress fields expressed in the transform domain are

$$
\begin{aligned}
& \bar{u}^{*}=p\left(\eta \bar{\phi}_{\mathrm{d}}^{*} \mathrm{e}^{p \gamma_{\mathrm{L}} y}+\gamma_{\mathrm{T}} \bar{\psi}_{\mathrm{d}}^{*} \mathrm{e}^{p \gamma_{\mathrm{T}} y}\right)+p\left(\eta \bar{\phi}_{\mathrm{u}}^{*} \mathrm{e}^{-p \gamma_{\mathrm{L}} y}-\gamma_{\mathrm{T}} \bar{\psi}_{\mathrm{u}}^{*} \mathrm{e}^{p \gamma_{\mathrm{T}} y}\right), \\
& \bar{v}^{*}=p\left(\gamma_{\mathrm{L}} \bar{\phi}_{\mathrm{d}}^{*} \mathrm{e}^{p \gamma_{\mathrm{L}} y}-\eta \overline{\mathrm{d}}_{\mathrm{d}}^{*} \mathrm{e}^{p \gamma_{\mathrm{T}} y}\right)+p\left(-\gamma_{\mathrm{L}} \bar{\phi}_{\mathrm{u}}^{*} \mathrm{e}^{-p \gamma_{\mathrm{L}} y}-\eta \bar{\psi}_{\mathrm{u}}^{*} \mathrm{e}^{p \gamma_{\mathrm{L}} y}\right), \\
& \bar{\sigma}_{x x}^{*}=\mu p^{2}\left[\left(s_{\mathrm{T}}^{2}-2 s_{\mathrm{L}}^{2}+2 \eta^{2}\right) \bar{\phi}_{\mathrm{d}}^{*} \mathrm{e}^{p \gamma_{\mathrm{L}} y}+2 \eta \gamma_{\mathrm{T}} \bar{\psi}_{\mathrm{d}}^{*} \mathrm{e}^{p \gamma_{\mathrm{T}} y}\right] \\
& \quad+\mu p^{2}\left[\left(s_{\mathrm{T}}^{2}-2 s_{\mathrm{L}}^{2}+2 \eta^{2}\right) \bar{\phi}_{\mathrm{u}}^{*} \mathrm{e}^{-p \gamma_{\mathrm{L}} y}-2 \eta \gamma_{\mathrm{T}} \bar{\psi}_{\mathrm{u}}^{*} \mathrm{e}^{-p \gamma_{\mathrm{T}} y}\right], \\
& \bar{\sigma}_{y y}^{*}=\mu p^{2}\left[\left(s_{\mathrm{T}}^{2}-2 \eta^{2}\right) \bar{\phi}_{\mathrm{d}}^{*} \mathrm{e}^{p \gamma_{\mathrm{L}} y}-2 \eta \gamma_{\mathrm{T}} \bar{\psi}_{\mathrm{d}}^{*} \mathrm{e}^{p \gamma_{\mathrm{T}} y}\right]+\mu p^{2}\left[\left(s_{\mathrm{T}}^{2}-2 \eta^{2}\right) \bar{\phi}_{\mathrm{u}}^{*} \mathrm{e}^{-p \gamma_{\mathrm{L}} y}+2 \eta \gamma_{\mathrm{T}} \bar{\psi}_{\mathrm{u}}^{*} \mathrm{e}^{-p \gamma_{\mathrm{T}} y}\right], \\
& \bar{\sigma}_{x y}^{*}=\mu p^{2}\left[2 \eta \gamma_{\mathrm{L}} \bar{\phi}_{\mathrm{d}}^{*} \mathrm{e}^{p \gamma_{\mathrm{L}} y}+\left(s_{\mathrm{T}}^{2}-2 \eta^{2}\right) \bar{\psi}_{\mathrm{d}}^{*} \mathrm{e}^{p \gamma_{\mathrm{T}} y}\right]+\mu p^{2}\left[-2 \eta \gamma_{\mathrm{L}} \bar{\phi}_{\mathrm{u}}^{*} \mathrm{e}^{-p \gamma_{\mathrm{L}} y}+\left(s_{\mathrm{T}}^{2}-2 \eta^{2}\right) \psi_{\mathrm{u}}^{*} \mathrm{e}^{-p \gamma_{\mathrm{T}} y}\right] .
\end{aligned}
$$

The displacement and stress fields are separated into two parts: one is contributed from the upward waves and the other is from the downward waves. The coefficients of $\bar{\phi}_{\mathrm{u}}^{*}, \psi_{\mathrm{u}}^{*}, \bar{\phi}_{\mathrm{d}}^{*}$, and $\bar{\psi}_{\mathrm{d}}^{*}$, in this expression are called the receiver functions by the theory of generalized ray.

For convenience, we define the traction vector on the $y$ plane as

$$
\hat{\tau}(y) \equiv\left(\begin{array}{c}
\bar{\sigma}_{y y}^{*}(y ; p, \eta) \\
\bar{\sigma}_{x y}^{*}(y ; p, \eta)
\end{array}\right)
$$

If we rearrange the unknown coefficients as two vectors $\mathbf{n}_{\mathrm{u}}$ and $\mathbf{n}_{\mathrm{d}}$ for the up-going and downgoing potentials, respectively, then we have,

$$
\hat{\mathbf{n}}_{\mathrm{u}}=\left(\begin{array}{l}
\bar{\phi}_{\mathrm{u}}^{*}(p, \eta) \\
\bar{\psi}_{\mathrm{u}}^{*}(p, \eta)
\end{array}\right),
$$

and

$$
\hat{\mathbf{n}}_{\mathrm{d}}=\left(\begin{array}{l}
\bar{\phi}_{\mathrm{d}}^{*}(p, \eta) \\
\bar{\psi}_{\mathrm{d}}^{*}(p, \eta)
\end{array}\right) .
$$

From (6d) and (6e), the traction vector $\hat{\tau}(y)$ becomes

$$
\hat{\tau}(y)=\mathbf{M}_{\mathrm{d}}(y) \hat{\mathbf{n}}_{\mathrm{d}}+\mathbf{M}_{\mathrm{u}}(y) \hat{\mathbf{n}}_{\mathrm{u}},
$$

where the $2 \times 2$ matrix $\mathbf{M}_{\mathrm{u}}$ and $\mathbf{M}_{\mathrm{d}}$ relate the up-going waves and down-going waves to the traction vector, and 


$$
\begin{aligned}
& \mathbf{M}_{\mathrm{d}}(y)=\mu p^{2}\left[\begin{array}{cc}
\left(s_{\mathrm{T}}^{2}-2 \eta^{2}\right) \mathrm{e}^{p \gamma_{\mathrm{L}} y} & -2 \eta \gamma_{\mathrm{T}} \mathrm{e}^{p \gamma_{\mathrm{T}} y} \\
2 \eta \gamma_{\mathrm{L}} \mathrm{e}^{p \gamma_{\mathrm{L}} y} & \left(s_{\mathrm{T}}^{2}-2 \eta^{2}\right) \mathrm{e}^{p \gamma_{\mathrm{T}} y}
\end{array}\right], \\
& \mathbf{M}_{\mathrm{u}}(y)=\mu p^{2}\left[\begin{array}{cc}
\left(s_{\mathrm{T}}^{2}-2 \eta^{2}\right) \mathrm{e}^{-p \gamma_{\mathrm{L}} y} & 2 \eta \gamma_{\mathrm{T}} \mathrm{e}^{-p \gamma_{\mathrm{T}} y} \\
-2 \eta \gamma_{\mathrm{L}} \mathrm{e}^{-p \gamma_{\mathrm{L}} y} & \left(s_{\mathrm{T}}^{2}-2 \eta^{2}\right) \mathrm{e}^{-p \gamma_{\mathrm{T}} y}
\end{array}\right] .
\end{aligned}
$$

\section{Transient solutions in the transform domain}

In this section, the method of constructing the solution in the Laplace transform domain is explained in detail. The solution is expressed as an infinite series of matrix in such a way that each term has its physical meaning and can be identified by the theory of generalized ray.

The solution for the problem described in the previous section can be obtained by determining the unknown coefficient vectors $\hat{\mathbf{n}}_{\mathrm{d}}$ and $\hat{\mathbf{n}}_{\mathrm{u}}$ from the boundary conditions in the transform domain. The boundary conditions described in (1) expressed in the Laplace transform domain are denoted as $\hat{\tau}_{1}$ and $\hat{\tau}_{2}$ for top and lower surface, respectively, where

$$
\hat{\tau}_{1}=\left(\begin{array}{l}
\bar{\sigma}_{y y}^{*}(0 ; p, \eta) \\
\bar{\sigma}_{x y}^{*}(0 ; p, \eta)
\end{array}\right)
$$

and

$$
\hat{\tau}_{2}=\left(\begin{array}{c}
\bar{\sigma}_{y y}^{*}(-h ; p, \eta) \\
\bar{\sigma}_{x y}^{*}(-h ; p, \eta)
\end{array}\right)
$$

With aids of (9), the as yet unknown vectors $\hat{\mathbf{n}}_{\mathrm{d}}$ and $\hat{\mathbf{n}}_{\mathrm{u}}$ in (8a) and (8b) can be determined from the boundary conditions at $y=0$ and $y=-h$, which give two matrix equations as

$$
\left[\begin{array}{c:c}
\mathbf{M}_{\mathrm{d}}(0) & \vdots \\
\cdots \cdots \cdots \cdots & \mathbf{M}_{\mathrm{u}}(0) \\
\mathbf{M}_{\mathrm{d}}(-h) & \cdots \cdots \cdots \\
\mathbf{M}_{\mathrm{u}}(-h)
\end{array}\right]\left(\begin{array}{c}
\hat{\mathbf{n}}_{\mathrm{d}} \\
\cdots \\
\hat{\mathbf{n}}_{\mathrm{u}}
\end{array}\right)=\left(\begin{array}{c}
\hat{\tau}_{1} \\
\cdots \\
\hat{\tau}_{2}
\end{array}\right),
$$

or in a more compact form,

$$
\mathbf{M n}=\hat{\mathbf{t}},
$$

where the unknown coefficients $\hat{\mathbf{n}}_{\mathrm{d}}$ and $\hat{\mathbf{n}}_{\mathrm{u}}$ are stacked up to form the vector $\hat{\mathbf{n}}$ and the boundary traction vector $\hat{\mathbf{t}}$ are formed of $\hat{\tau}_{1}$ and $\hat{\tau}_{2}$. The stacked matrix equation as shown in (13) can be solved directly by means of multiplying both sides of the matrix equation by inverting matrix of coefficient matrix or by Cramer's rules,

$$
\hat{\mathbf{n}}=\mathbf{M}^{-1} \hat{\mathbf{t}} \text {. }
$$

With the Laplace transform solution at hand, we should perform the inverse transform to get the transient solution in time domain. The inversion of Laplace transform usually involves a summation of residues which are infinite in number, hence, the accuracy of the final answer depends on the number of terms taken in the series. As an alternative way to solve the problem, the inverse 
transform can be also accomplished with Cagniard's method by expanding the denominator of the coefficients $\bar{\phi}_{\mathrm{u}}^{*}, \bar{\phi}_{\mathrm{d}}^{*}, \bar{\psi}_{\mathrm{u}}^{*}$, and $\bar{\psi}_{\mathrm{d}}^{*}$ into a series of the exponential components but only the first few terms were constructed in the formal expansion proposed by Mencher (1953). Each term in the series is corresponding to a generalized ray. We will proposed a new methodology to construct the solution of the problem in a more compact form in Section 3.1.

\subsection{New methodology in the matrix formulation}

We rewrite the coefficient matrix $\mathbf{M}$ in (13) to an alternative form

$$
\mathbf{M}=\mathbf{D}+\mathbf{L}+\mathbf{U}=\mathbf{D}\left[\mathbf{I}+\mathbf{D}^{-1}(\mathbf{L}+\mathbf{U})\right],
$$

where the matrix $\mathbf{D}, \mathbf{L}, \mathbf{U}$ are the diagonal, lower triangle, and upper triangle parts of the coefficient matrix $\mathbf{M}$ and are expressed as follows

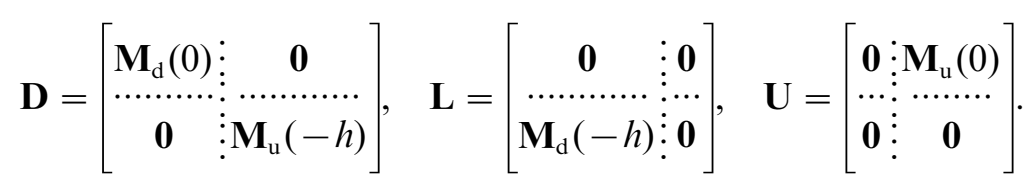

The unknown coefficient vector $\hat{\mathbf{n}}$ is then expressed in the following form

$$
\hat{\mathbf{n}}=\left[\mathbf{I}+\mathbf{D}^{-1}(\mathbf{L}+\mathbf{U})\right]^{-1}\left(\mathbf{D}^{-1} \hat{\mathbf{t}}\right)=[\mathbf{I}-\mathbf{R}]^{-1} \hat{\mathbf{s}},
$$

in which

$$
\mathbf{R}=-\mathbf{D}^{-1}(\mathbf{L}+\mathbf{U})
$$

and

$$
\hat{\mathbf{s}}=\mathbf{D}^{-1} \hat{\mathbf{t}}
$$

The vector $\hat{\mathbf{s}}$ is expressed in the following form

$$
\hat{\mathbf{s}}=\left(\begin{array}{l}
\hat{\mathbf{s}}_{\mathrm{d}} \\
\hat{\mathbf{s}}_{\mathrm{u}}
\end{array}\right)=\left(\begin{array}{c}
\mathbf{M}_{\mathrm{d}}^{-1}(0) \hat{\tau}_{1} \\
\mathbf{M}_{\mathrm{u}}^{-1}(-h) \hat{\tau}_{2}
\end{array}\right) .
$$

The matrix $\mathbf{R}$ is represented as

$$
\mathbf{R}=\left[\begin{array}{c:c}
\mathbf{0} & \vdots \\
\mathbf{R} \\
\cdots & \cdots \\
\mathbf{R}_{\mathrm{u}} & \vdots \mathbf{0}
\end{array}\right]
$$

where

$$
\mathbf{R}_{\mathrm{d}}=-\mathbf{M}_{\mathrm{d}}^{-1}(0) \mathbf{M}_{\mathrm{u}}(0)=\left[\begin{array}{cc}
R_{\mathrm{pp}} & R_{\mathrm{sp}} \\
R_{\mathrm{ps}} & R_{\mathrm{ss}}
\end{array}\right],
$$

and 


$$
\mathbf{R}_{\mathrm{u}}=-\mathbf{M}_{\mathrm{u}}^{-1}(-h) \mathbf{M}_{\mathrm{d}}(-h)=\left[\begin{array}{cc}
R^{\mathrm{pp}} \mathrm{e}^{-2 p \gamma_{\mathrm{L}} h} & R^{\mathrm{sp}} \mathrm{e}^{-p\left(\gamma_{\mathrm{L}}+\gamma_{\mathrm{T}}\right) h} \\
R^{\mathrm{ps}} \mathrm{e}^{-p\left(\gamma_{\mathrm{L}}+\gamma_{\mathrm{T}}\right) h} & R^{\mathrm{ss}} \mathrm{e}^{-2 p \gamma_{\mathrm{T}} h}
\end{array}\right] .
$$

The elements in matrix $\mathbf{R}_{\mathrm{d}}$ and $\mathbf{R}_{\mathrm{u}}$ are

$$
\begin{aligned}
& R^{\mathrm{pp}}=R^{\mathrm{ss}}=R_{\mathrm{pp}}=R_{\mathrm{ss}}=\left[4 \eta^{2} \gamma_{\mathrm{L}} \gamma_{\mathrm{T}}-\left(s_{\mathrm{T}}^{2}-2 \eta^{2}\right)^{2}\right] / R(\eta), \\
& R^{\mathrm{ps}}=-R_{\mathrm{ps}}=-4 \eta \gamma_{\mathrm{L}}\left(s_{\mathrm{T}}^{2}-2 \eta^{2}\right) / R(\eta), \\
& R^{\mathrm{sp}}=-R_{\mathrm{sp}}=4 \eta \gamma_{\mathrm{T}}\left(s_{\mathrm{T}}^{2}-2 \eta^{2}\right) / R(\eta),
\end{aligned}
$$

and

$$
R(\eta)=\left[\left(s_{\mathrm{T}}^{2}-2 \eta^{2}\right)^{2}+4 \eta^{2} \gamma_{\mathrm{L}} \gamma_{\mathrm{T}}\right]
$$

in which $R(\eta)$ is the well-known Rayleigh wave equation. The elements of matrix $\mathbf{R}$ are exactly the same as the reflection coefficients for plane waves interaction with traction free boundary with phase changes.

By expanding the matrix $(\mathbf{I}-\mathbf{R})^{-1}$ into a power series of matrix $\mathbf{R}$, the unknown coefficient vector $\hat{\mathbf{n}}$ can be rewritten as

$$
\hat{\mathbf{n}}=(\mathbf{I}-\mathbf{R})^{-1} \hat{\mathbf{s}}=\sum_{i=0}^{\infty} \mathbf{R} \hat{\mathbf{s}}
$$

where $\hat{\mathbf{s}}$ and $\mathbf{R}$ are given in (20) and (21). It is noted that the sum of absolute value of all elements in each row of matrix $\mathbf{R}$ is less than or equal to unity, hence all the eigenvalues of matrix $\mathbf{R}$ are allocated in a unit circle in the complex plane and the convergence of the series is expected. The expansion is applicable to practical problems since only the first few terms out of the infinite sum are relevant for any given time of interest.

In (23), the matrix $\mathbf{R}$ characterizes the multiple reflections of all waves within the strip, and the vector $\hat{\mathbf{s}}$ specifies the source waves generated by the applied traction at two lateral surfaces. The physical meaning of the $\mathbf{R}$ matrix will be clear by considering the characteristic of the waves interacting with the a planar boundary, which will be discussed next.

\subsection{Waves in the strip}

The transient wave field in a strip can be decomposed into many groups of waves in a stepwise fashion by disregarding the top surface $y=0$ and lower surface $y=-h$ in turn. The physical meaning of solution expressed in (23) will be clear after the derivation by the following approach. The traction applied on the top surface of the strip will be considered first and the solution for the traction applied on the lower surface can be constructed in a similar manner. The complete solution of the problem is obtained by superposing of all groups of waves which are generated by the tractions applied on both surfaces.

A case in which the top surface is loaded and the lower surface remains free is considered first. To begin with, the problem related to a half-plane which is subjected to the same load as that applied to the top surface should be worked out. The applied traction on the top surface generates 
a set of waves propagating along the $-y$ direction. The wave potentials $\hat{\mathbf{n}}_{\mathrm{d} 0}$ representing the wave in the semi-infinite domain can be obtained from the boundary condition at $y=0$

$$
\hat{\mathbf{n}}_{\mathrm{d} 0}=\mathbf{M}_{\mathrm{d}}^{-1}(0) \hat{\tau}_{1} \equiv \hat{\mathbf{s}}_{\mathrm{d}}
$$

where the subscript 0 indicates the zeroth order of reflection and $\hat{\mathbf{n}}_{\mathrm{d} 0}$ is denoted as $\hat{\mathbf{s}}_{\mathrm{d}}$ hereinafter to represent the source waves produced by the applied force. The source wave potential $\hat{\mathbf{s}}_{\mathrm{d}}$ can be considered as the incident waves to the lower surface $y=-h$ and a group of waves represented by $\hat{\mathbf{n}}_{\mathrm{u} 1}$ will be induced by applying an opposite traction on the lower surface. The traction-free boundary condition at $y=-h$ yields

$$
\mathbf{M}_{\mathrm{d}}(-h) \hat{\mathbf{s}}_{\mathrm{d}}+\mathbf{M}_{\mathrm{u}}(-h) \hat{\mathbf{n}}_{\mathrm{u} 1}=\mathbf{0},
$$

then $\hat{\mathbf{n}}_{\mathrm{u} 1}$ is expressed in terms of the incident wave $\hat{\mathbf{s}}_{\mathrm{d}}$ by

$$
\hat{\mathbf{n}}_{\mathrm{u} 1}=\mathbf{R}_{\mathrm{u}} \hat{\mathbf{s}}_{\mathrm{d}}
$$

here $\mathbf{R}_{\mathrm{u}}$ is exactly the same as that given in (21b). The physical meaning of matrix $\mathbf{R}_{\mathrm{u}}$ now makes clear that each element of the matrix represents a reflection coefficient of the lower surface of the strip with phase change.

The matrix $\mathbf{R}_{\mathrm{u}}$ characterizes the transfer relation between the incident and the reflected wave potentials. If there is a pressure wave propagating toward the free surface $y=-h$, the coefficient of reflected pressure waves can be obtained immediately by multiplying the coefficient of incident pressure waves with $\left[\mathbf{R}_{\mathrm{u}}\right]_{11}$. The coefficient of the reflected shear wave can be obtained from the incident coefficient by multiplying $\left[\mathbf{R}_{\mathrm{u}}\right]_{12}$. The two reflected waves generated from the incident pressure wave are denoted as pp- and ps-waves. Similarly, we denote reflected sp- and ss-waves from incident shear waves and the coefficients of these reflected waves can be obtained by multiplying the coefficient of incident shear wave with $\left[\mathbf{R}_{\mathrm{u}}\right]_{21}$ and $\left[\mathbf{R}_{\mathrm{u}}\right]_{22}$, respectively.

After some later time, these reflected waves (i.e. $\hat{\mathbf{n}}_{\mathrm{u} 1}$ ) will reach the top surface and another group of waves $\hat{\mathbf{n}}_{\mathrm{d} 2}$ is generated to cancel the traction produced by $\hat{\mathbf{n}}_{\mathrm{u} 1}$ at $y=0$, we have

$$
\mathbf{M}_{\mathrm{d}}(0) \hat{\mathbf{n}}_{\mathrm{d} 2}+\mathbf{M}_{\mathrm{u}}(0) \hat{\mathbf{n}}_{\mathrm{u} 1}=\mathbf{0},
$$

and $\hat{\mathbf{n}}_{\mathrm{d} 2}$ is given by

$$
\hat{\mathbf{n}}_{\mathrm{d} 2}=\mathbf{R}_{\mathrm{d}} \hat{\mathbf{n}}_{\mathrm{u} 1}=\mathbf{R}_{\mathrm{d}} \mathbf{R}_{\mathrm{u}} \hat{\mathbf{s}}_{\mathrm{d}}
$$

where $\mathbf{R}_{\mathrm{d}}$ is given in (21a). Hence, any group of reflected waves propagating downward can be found by simply multiplying the reflection matrix $\mathbf{R}_{\mathrm{d}}$ with the incident wave $\hat{\mathbf{n}}_{\mathrm{u}}$.

By satisfying the two boundary conditions alternatively, the waves in the strip are decomposed into many groups of waves with different reflection orders. There are infinitely many groups of waves in the strip that will be generated. A synthesis of all the wave groups leads to the total wave field in the strip. The complete solution for the traction applied on the top surface is then given by

$$
\hat{\mathbf{n}}_{\mathrm{d}}=\sum_{i=0}^{\infty}\left(\mathbf{R}_{\mathrm{d}} \mathbf{R}_{\mathrm{u}}\right)^{i} \hat{\mathbf{s}}_{\mathrm{d}}
$$

and 


$$
\hat{\mathbf{n}}_{\mathrm{u}}=\sum_{i=0}^{\infty} \mathbf{R}_{\mathrm{u}}\left(\mathbf{R}_{\mathrm{d}} \mathbf{R}_{\mathrm{u}}\right)^{i} \hat{\mathbf{s}}_{\mathrm{d}}
$$

Each of the infinitely many terms expressed in (29), except the first one, i.e. $\hat{\mathbf{s}}_{\mathrm{d}}$, is due to applying an external load in order to satisfy the traction free condition at surfaces $y=0$ and $y=-h$. For any given time of interest, only a few terms out of the infinite sum are needed and the higher order terms in the series begin to affect the solution after longer periods of time. The solution can be rewritten in a more concise form as

$$
\left(\begin{array}{c}
\hat{\mathbf{n}}_{\mathrm{d}} \\
\cdots \\
\hat{\mathbf{n}}_{\mathrm{u}}
\end{array}\right)=\sum_{i=0}^{\infty}\left[\begin{array}{ccc}
\mathbf{0} & \vdots & \mathbf{R}_{\mathrm{d}} \\
\cdots & \cdots \\
\mathbf{R}_{\mathrm{u}} & \vdots & \mathbf{0}
\end{array}\right]^{i}\left(\begin{array}{c}
\hat{\mathbf{s}}_{\mathrm{d}} \\
\cdots \\
\mathbf{0}
\end{array}\right) .
$$

The solution for the tractions applied on the lower surface can be derived in a similar manner. The complete solution of the strip for applying traction on both surfaces can then be constructed easily and expressed as follows

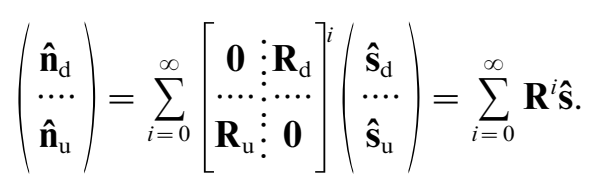

The solution is exactly the same as that given in (23). The term $\mathbf{R}$ is represents a group of waves which is reflected by both surfaces of the strip $i$ times. It is worthy to note that each wave diverges in the long-time limit but the sum of all the waves in each group goes to a static value when the applied force tends to a static force as time increases. Each wave within a group of the same reflection order can be identified by the generalized ray method.

It is shown in this method that whenever waves reach any one of the two surfaces, a half-space kind of solution is added to them. The complete solution consists of the sum of infinitely many contributions, each of which corresponds to a group of reflection waves generated from one of the surfaces. Since each term in the infinite sum is derived from a half-space kind of solution, hence Cagniard's method of Laplace inversion can be applied directly. Although the solution for waves in a strip can be constructed in Section 3.2, the method provided in Section 3.1 is more simple and elegant.

\subsection{The ray tracing technique}

Figure 2 shows that the total response of a strip with a dynamic loading applied on its top surface is decomposed into infinite wave groups each containing many reflected waves. There are $2^{i+1}$ waves in the $i$ th group that reflected $i$ times between the top and lower surface of the strip. Because of the mode conversion, we denote each reflected wave by attaching the mode of the source wave to the mode of the reflected wave, such as pps or pspspp, to distinguish the mode conversion at each step of reflection as shown in Fig. 2. The waves in the $i$ th group are coded by $i+1$ letters, and each letter can be either a $\mathrm{p}$ or $\mathrm{s}$ mode. The last letter of this coding system represents the propagating mode of the wave and the remainder designates the history of this 


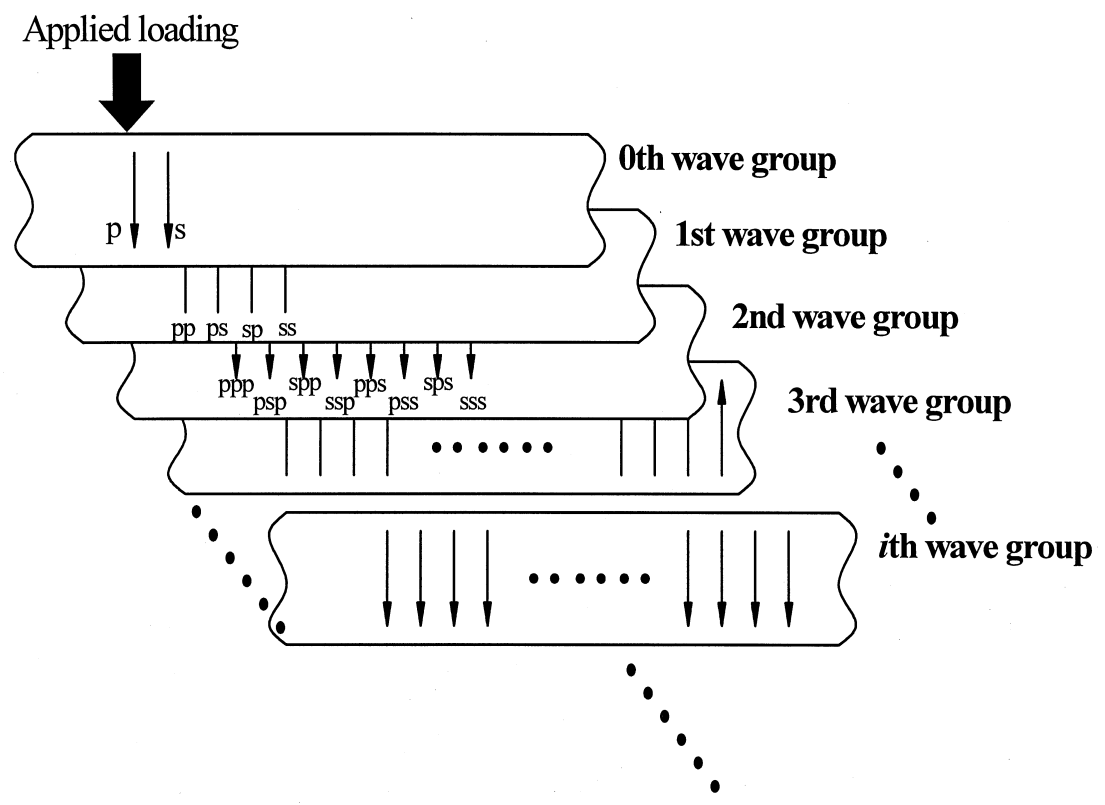

Fig. 2. The classification of reflected waves in a strip.

wave. We can distinguish all the waves and sum them up to obtain the total response for the numerical calculation with aids of the coding system.

Each wave can be represented by a set of number $(i, j, k)$ in which the first number $i$ ranging from 0 to infinity is the number of reflection for this wave, the second number $j$ ranging from 0 $2^{i}-1$ stores the historical information of the wave and the third number $k$ indicates the mode of the wave propagating in the strip. The set of number $(i, j, k)$ can be transformed to the coding system in Fig. 2. The complete solution is the sum of all the waves generated by the sources. We sort the potentials, $\bar{\phi}^{*}(k=0)$ or $\bar{\psi}^{*}(k=1)$, into a series $\bar{E}^{*}$ which mainly contains two parts,

$$
\bar{E}^{*}(p, \eta)=\bar{E}_{+}^{*}+\bar{E}_{-}^{*},
$$

in which

$$
\bar{E}_{ \pm}^{*}(p, \eta)=\sum_{i=0}^{\infty} \sum_{j=0}^{2^{i}-1} \sum_{k=0}^{1} \bar{E}_{ \pm(i, j, k)}^{*}
$$

where

$$
\bar{E}_{ \pm(i, j, k)}^{*}=\prod_{l=1}^{i} G_{l}(\eta) S_{ \pm}(p, \eta) \mathrm{e}^{-p\left[\left(m \gamma_{\mathrm{L}}+n \gamma_{\mathrm{T}}\right) h \pm(-1)^{i} \gamma_{\mathrm{T}, \mathrm{L}} y\right]},
$$

the sign in the subscript is chosen positive when the source is on the top surface and minus when the source is on the lower surface. $S(p, \eta)$ denotes the source function which is chosen from the elements of source vector $\hat{\mathbf{s}}$ in (20) and is dependent on the source of the corresponding wave. The symbol $\gamma_{\mathrm{T}, \mathrm{L}}$, which related to the propagating mode of the wave, is selected as $\gamma_{\mathrm{T}}$ when $k=1$, and 
as $\gamma_{\mathrm{L}}$ when $k=0$. The $m$ and $n$ in the phase function as well as the product of reflection coefficients $\Pi G_{l}(\eta)$ can be determined by tracing the origin of the wave and can be easily found by multiplying the corresponding generalized reflection coefficient with phase shift $[\mathbf{R}]$ expressed in (21) at each step of reflection.

So far, we have constructed the solution of displacement potentials in the transform domain with matrix formula. The displacements and stresses can be obtained by substituting the displacement potential into (6). The remaining task is to evaluate the inverse transform of these expressions. The inverse transform is based on the well-known Cagniard method. The details of Cagniard's method are discussed by Cagniard (1939) and Pao and Gajewski (1977) and will be illustrated by an example indicated in the next section.

\section{Transient solution for an infinite strip subjected to a normal loading}

We have formulated the two-dimensional solution in Laplace transform domain for a strip subjected to a distributed loading with arbitrary time dependence in the previous section. Now we consider in detail for the problem of a vertical concentrated force with arbitrary time dependence acting on the top surface at $x=0, y=0$. The boundary conditions on top and lower surfaces are expressed as

$$
\left\{\begin{array} { l } 
{ \sigma _ { x y } ( x , 0 , t ) = 0 } \\
{ \sigma _ { y y } ( x , 0 , t ) = \sigma _ { 0 } \delta ( x ) f ( t ) }
\end{array} \quad \left\{\begin{array}{l}
\sigma_{x y}(x,-h, t)=0 \\
\sigma_{y y}(x,-h, t)=0
\end{array} \text { for }-\infty \leqslant x \leqslant \infty .\right.\right.
$$

Since there are no sources on the lower surface, only the downward waves will be generated from the applied loading. For convenience, the source function can be factored into two functions, one function depends on $\eta$ and the other on $p$ as follows

$$
\hat{\mathbf{s}}_{\mathrm{d}}=\mathbf{M}_{\mathrm{d}}^{-1}(0) \hat{\tau}_{1}=\bar{L}(p)\left(\begin{array}{c}
S_{\mathrm{p}} \\
S_{\mathrm{s}}
\end{array}\right), \quad \hat{\mathbf{s}}_{\mathrm{u}}=\mathbf{0}
$$

where

$$
\bar{L}(p)=\frac{\sigma_{0}}{\mu} \frac{\bar{f}(p)}{p^{2}}
$$

and

$$
\left(\begin{array}{c}
S_{\mathrm{p}} \\
S_{\mathrm{s}}
\end{array}\right)=\frac{1}{R(\eta)}\left(\begin{array}{c}
-\left(s_{\mathrm{T}}^{2}-2 \eta^{2}\right) \\
2 \eta \gamma_{\mathrm{L}}
\end{array}\right)
$$

To perform Cagniard's method, each individual wave can be rewritten as follows

$$
\bar{E}_{-(i, j, k)}^{*}(p, \eta)=\bar{L}(p) \bar{K}_{-(i, j, k)}^{*}(\eta) \mathrm{e}^{-p\left[\left(m \gamma_{\mathrm{L}}+m \gamma_{\mathrm{T}}\right) h-(-1)^{i} \gamma_{k} y\right]},
$$

where 


$$
\bar{K}_{-(i, j, k)}^{*}(\eta)=\prod_{l=1}^{i} G_{l}(\eta) S_{-}(\eta)
$$

The inverse formula for the two-sided Laplace transform of this general expression leads to

$$
\bar{E}_{-(i, j, k)}(p)=\frac{p \bar{L}(p)}{2 \pi i} \int_{\eta_{1}-i \infty}^{\eta_{1}+i \infty} \bar{K}_{-(i, j, k)}^{*}(\eta) \mathrm{e}^{-p\left[\left(m \gamma_{\mathrm{L}}+m \gamma_{\mathrm{T}}\right) h-(-1)^{i} \gamma_{k} y-\eta x\right]} \mathrm{d} \eta
$$

The overbar symbol is used to denote the transform on time $t$.

The exact transient solution in time domain can then be found by the application of Cagniard's method. The idea of the method is to deform the path of integration in the complex $\eta$-plane along a new path in such a manner that the inverse Laplace transform of the integral along the new path of integration can be obtained by inspection. The desired path of integration in the complex $\eta$ plane is defined by the following equation

$$
t=\left[\left(m \gamma_{\mathrm{L}}+n \gamma_{\mathrm{T}}\right) h-(-1)^{i} \gamma_{\mathrm{T}, \mathrm{L}} y-\eta x\right] .
$$

The root of the equation for p-wave $(k=0)$ is denoted as $\eta_{\mathrm{p}}$ and for s-wave $(k=1)$ as $\eta_{\mathrm{s}}$. To obtain the desired path, it is convenient to find the root of (37) numerically by applying the modified Newton's method. Excellent numerical solution can be found by four or five iterations.

The arrival time of each multi-reflected wave is determined from the stationary value of $t$, which is a function of $\eta$. The global stationary value for $t$ is determined by the condition

$$
\frac{\mathrm{d} t}{\mathrm{~d} \eta}=0
$$

From (37) and (38), the arrival time of the multi-reflected wave can be determined numerically. The corresponding arrival time is denoted as $t_{\mathrm{p}}$ and $t_{\mathrm{s}}$ for pressure and shear wave, respectively. In addition, there are branch points in the complex $\eta$ plane. To deform the Bromwich contour into Cagniard's path, an additional head wave will be induced when Cagniard's path encloses the branch point. An additional contribution will be generated from the deformed path around the branch cut. The contribution of head wave acts between $t_{\mathrm{h}}$ and $t_{\mathrm{s}}$, where $t_{\mathrm{h}}$ denote the arrival time of the head wave that is found by substituting $\eta$ with $-s_{\mathrm{L}}$ in (37) when Cagniard's path encloses the branch point $-s_{\mathrm{L}}$.

Comparing (36) with the Laplace transform formulation, the inverse Laplace transform can be achieved by using the convolution theorem for Laplace transform. For example, the transient solution for a p-wave typed potential $E_{-(i, j, k)}(k=0)$ can be expressed as follows

$$
E_{-(i, j, 0)}(x, y, t)=\frac{1}{\pi} \mathscr{L}^{-1}\{p \bar{L}(p)\} * \operatorname{Im}\left\{\bar{K}_{-(i, j, 0)}^{*} \frac{\mathrm{d} \eta}{\mathrm{d} t} H\left(t-t_{\mathrm{p}}\right)\right\}_{\eta=\eta_{\mathrm{p}}},
$$

where $H\left(t-t_{\mathrm{p}}\right)$ is the unit step time function. Finally, transient solutions for displacements and stresses can be found and are expressed as follows: 


$$
\begin{aligned}
& u=\frac{1}{\pi} \mathscr{L}^{-1}\left\{p^{2} \bar{L}(p)\right\} * \sum_{i=0}^{\infty} \sum_{j=0}^{2^{i}-1}\left\{\begin{array}{l}
\operatorname{Im}\left\{\eta_{\mathrm{L}} \bar{K}_{-(i, j, 0)}^{*} \frac{\mathrm{d} \eta_{\mathrm{p}}}{\mathrm{d} t}\right\} H\left(t-t_{\mathrm{p}}\right) \\
+\operatorname{Im}\left\{(-1)^{i} \gamma_{\mathrm{T}} \bar{K}_{-(i, j, 1)}^{*} \frac{\mathrm{d} \eta_{\mathrm{s}}}{\mathrm{d} t}\right\} H\left(t-t_{\mathrm{s}}\right) \\
+\operatorname{Im}\left\{(-1)^{i} \gamma_{\mathrm{T}} \bar{K}_{-(i, j, 1)}^{*} \frac{\mathrm{d} \eta_{\mathrm{s}}}{\mathrm{d} t}\right\} H\left(\eta_{\mathrm{s}}+s_{\mathrm{L}}\right) H\left(t-t_{\mathrm{h}}\right) H\left(t_{\mathrm{s}}-t\right)
\end{array}\right\} \\
& v=\frac{1}{\pi} \mathscr{L}^{-1}\left\{p^{2} \bar{L}(p)\right\} * \sum_{i=0}^{\infty} \sum_{j=0}^{2^{i}-1}\left\{\begin{array}{l}
\operatorname{Im}\left\{(-1)^{i} \gamma_{\mathrm{L}} \bar{K}_{-(i, j, 0)}^{*} \frac{\mathrm{d} \eta_{\mathrm{p}}}{\mathrm{d} t}\right\} H\left(t-t_{\mathrm{p}}\right) \\
+\operatorname{Im}\left\{-\eta_{\mathrm{s}} \bar{K}_{-(i, j, 1)}^{*} \frac{\mathrm{d} \eta_{\mathrm{s}}}{\mathrm{d} t}\right\} H\left(t-t_{\mathrm{s}}\right) \\
+\operatorname{Im}\left\{-\eta_{\mathrm{s}} \bar{K}_{-(i, j, 1)}^{*} \frac{\mathrm{d} \eta_{\mathrm{s}}}{\mathrm{d} t}\right\} H\left(\eta_{\mathrm{h}}+s_{\mathrm{L}}\right) H\left(t-t_{\mathrm{h}}\right) H\left(t_{\mathrm{s}}-t\right)
\end{array}\right\}
\end{aligned}
$$

(40b)

$$
\sigma_{x x}=\frac{1}{\pi} \mathscr{L}^{-1}\left\{\mu p^{3} \bar{L}(p)\right\}
$$

$$
* \sum_{i=0}^{\infty} \sum_{j=0}^{2^{i}-1}\left\{\begin{array}{l}
\operatorname{Im}\left\{\left(s_{\mathrm{T}}^{2}-2 s_{\mathrm{L}}^{2}-2 \eta_{\mathrm{p}}^{2}\right) \bar{K}_{-(i, j, 0)}^{*} \frac{\mathrm{d} \eta_{\mathrm{p}}}{\mathrm{d} t}\right\} H\left(t-t_{\mathrm{p}}\right) \\
+\operatorname{Im}\left\{(-1)^{i} 2 \eta_{\mathrm{s}} \gamma_{\mathrm{T}} \bar{K}_{-(i, j, 1)}^{*} \frac{\mathrm{d} \eta_{\mathrm{s}}}{\mathrm{d} t}\right\} H\left(t-t_{\mathrm{s}}\right) \\
+\operatorname{Im}\left\{(-1)^{i} 2 \eta_{\mathrm{s}} \gamma_{\mathrm{T}} \bar{K}_{-(i, j, 1)}^{*} \frac{\mathrm{d} \eta_{\mathrm{s}}}{\mathrm{d} t}\right\} H\left(\eta_{\mathrm{s}}+s_{\mathrm{L}}\right) H\left(t-t_{\mathrm{h}}\right) H\left(t_{\mathrm{s}}-t\right)
\end{array}\right\}
$$

$$
\sigma_{y y}=\frac{1}{\pi} \mathscr{L}^{-1}\left\{\mu p^{3} L(p)\right\}
$$

$$
* \sum_{i=0}^{\infty} \sum_{j=0}^{2^{i}-1}\left\{\begin{array}{l}
\operatorname{Im}\left\{\left(s_{\mathrm{T}}^{2}-2 \eta_{\mathrm{p}}^{2}\right) \bar{K}_{-(i, j, 0)}^{*} \frac{\mathrm{d} \eta_{\mathrm{p}}}{\mathrm{d} t}\right\} H\left(t-t_{\mathrm{p}}\right) \\
+\operatorname{Im}\left\{-(-1)^{i} 2 \eta_{\mathrm{s}} \gamma_{\mathrm{T}} \bar{K}_{-(i, j, 1)}^{*} \frac{\mathrm{d} \eta_{\mathrm{s}}}{\mathrm{d} t}\right\} H\left(t-t_{\mathrm{s}}\right) \\
+\operatorname{Im}\left\{-(-1)^{i} 2 \eta_{\mathrm{s}} \gamma_{\mathrm{T}} \bar{K}_{-(i, j, 1)}^{*} \frac{\mathrm{d} \eta_{\mathrm{s}}}{\mathrm{d} t}\right\} H\left(\eta_{\mathrm{s}}+s_{\mathrm{L}}\right) H\left(t-t_{\mathrm{h}}\right) H\left(t_{\mathrm{s}}-t\right)
\end{array}\right\}
$$

$\sigma_{x y}=\frac{1}{\pi} \mathscr{L}^{-1}\left\{\mu p^{3} \bar{L}(p)\right\}$ 


$$
* \sum_{i=0}^{\infty} \sum_{j=0}^{2^{i}-1}\left\{\begin{array}{l}
\operatorname{Im}\left\{(-1)^{i} 2 \eta_{\mathrm{p}} \gamma_{\mathrm{T}} \bar{K}_{-(i, j, 0)}^{*} \frac{\mathrm{d} \eta_{\mathrm{p}}}{\mathrm{d} t}\right\} H\left(t-t_{\mathrm{p}}\right) \\
+\operatorname{Im}\left\{\left(s_{\mathrm{T}}^{2}-2 \eta_{\mathrm{s}}^{2}\right) \bar{K}_{-(i, j, 1)}^{*} \frac{\mathrm{d} \eta_{\mathrm{s}}}{\mathrm{d} t}\right\} H\left(t-t_{\mathrm{s}}\right) \\
+\operatorname{Im}\left\{\left(s_{\mathrm{T}}^{2}-2 \eta_{\mathrm{s}}^{2}\right) \bar{K}_{-(i, j, 1)}^{*} \frac{\mathrm{d} \eta_{\mathrm{s}}}{\mathrm{d} t}\right\} H\left(\eta_{\mathrm{s}}+s_{\mathrm{L}}\right) H\left(t-t_{\mathrm{h}}\right) H\left(t_{\mathrm{s}}-t\right)
\end{array}\right\},
$$

where the symbol $\mathscr{L}^{-1}\{\cdot\}$ denotes the inverse Laplace transform in $t$, and $*$ means the convolution of two functions with respect to time $t$.

\section{Numerical and experimental results}

The transient response of a strip subjected to a dynamic concentrated normal force on the top surface is formulated in detail in the previous section. In this section, the source time function $f(t)$ is selected as a Heaviside function for numerical calculations and experimental measurements. We set, in (33),

$$
f(t)=H(t),
$$

where $H(t)$ is the step function in time, which results

$$
L(p)=\frac{\sigma_{0}}{\mu} \frac{1}{p^{3}} .
$$

The wave front for the early time, depicted in Fig. 3, is constructed by connecting all the points of the same arrival time with aids of (37) and (38) for each multi-reflected wave in the strip. Figure 3 shows the wave fronts in the strip at different normalized time, $t / h s_{\mathrm{L}}=1.05,2.1,3.15,4.2$ and 5.25. The Poisson's ratio $v=0.34$ is chosen so that the p-wave is about twice that of the speed of the s-wave. The cylindrical p- and s-waves are generated by the point force. The planar head wave which is propagating along the free surface with $p$-wave speed and then emerging into the strip with s-wave speed is also generated in the mean time. As the time increases, the number of waves in the strip increases dramatically because of the mode conversion as the wave interacts with the boundary. Note that only waves without mode conversions (i.e. p-wave, s-wave, pp-wave, ss-wave, etc.) are cylindrical waves. The wave front of a wave with mode conversion (for example, pspwave) is no longer cylindrical. The head waves, which are straight lines, are also shown in the figure.

The transient responses of the strip are determined by summing all the contribution of reflected waves and the solution is exact up to the arrival of the next wave. There are at most twelve groups of waves (about 1500 waves) are considered for the numerical calculation of stresses and displacements. The time history of the transient stress $\sigma_{y y}$ at the epicenter point $(0,-h / 2)$, that oscillates periodically with slight damping at a later time, is shown in Fig. 4. The responses of stresses $\sigma_{y y}, \sigma_{x x}$ and $\sigma_{x y}$ at $(h,-h / 2)$ are shown in Figs 5-7. It is worth noting that the magnitude of the shear stress $\sigma_{x y}$ is much larger than that of normal stresses $\sigma_{y y}$ and $\sigma_{x x}$ at $(h,-h / 2)$, and the 


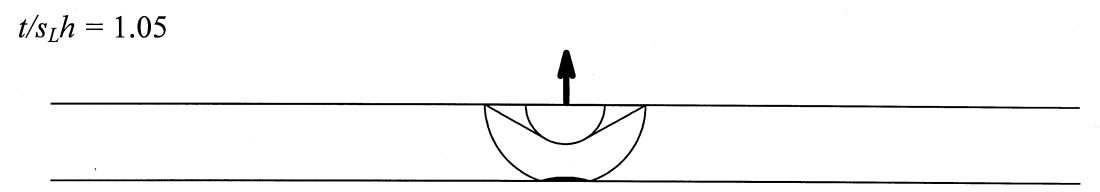

$t / s_{L} h=2.10$

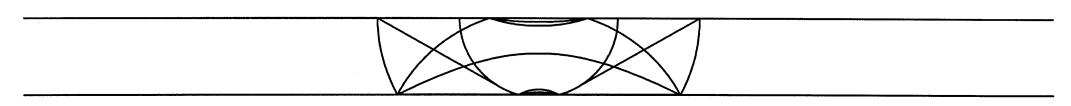

$t / s_{L} h=3.15$

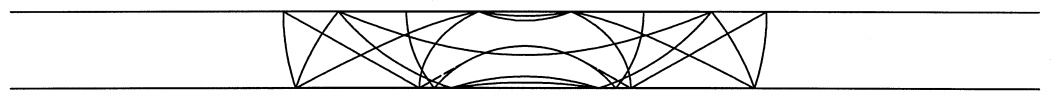

$t / s_{L} h=4.20$

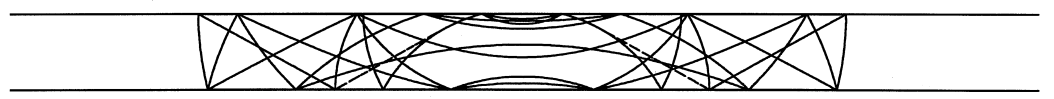

$t / s_{L} h=5.25$

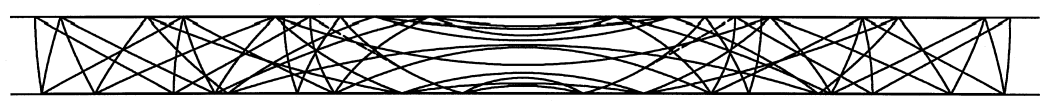

Fig. 3. Wave fronts for the early time response of a strip subjected to a concentrated loading applied at the top surface $(v=0.34)$.

magnitude of normal stresses are nearly zero. This phenomenon can also be expected from the static analysis. The stress field shows a square root singularity at the arrivals of all the reflected waves because of the Heaviside source time function. The singularity can be reduced by introducing the rounded-shoulder Heaviside source time function as shown in Fig. 8. The arising time of the source time function in each case is chosen as $2 \Delta=1.0,0.5$ and 0.1 . We can see that when the arising time is larger, the waveform is smoother.

It is interesting to note that each term in (40) diverges as time tends to infinity but the sum goes to a static value if the applied force is Heaviside or rounded-shoulder Heaviside time dependence. Furthermore, the transient response can be decomposed into many convergent subgroups in which the waves of the same number of reflection are summed. As an illustration, the time history for stress $\sigma_{y y}$ at $(h,-h / 2)$ for the contribution from each group is plotted in Fig. 9. There are $2^{i+1}$ waves in the $i$ th group and the horizontal line denotes the corresponding static value of each group. The static value can be obtained by using the Airy stress function and a similar scheme as in this study. We can see that each group of waves converge to the corresponding static value as time is large. 


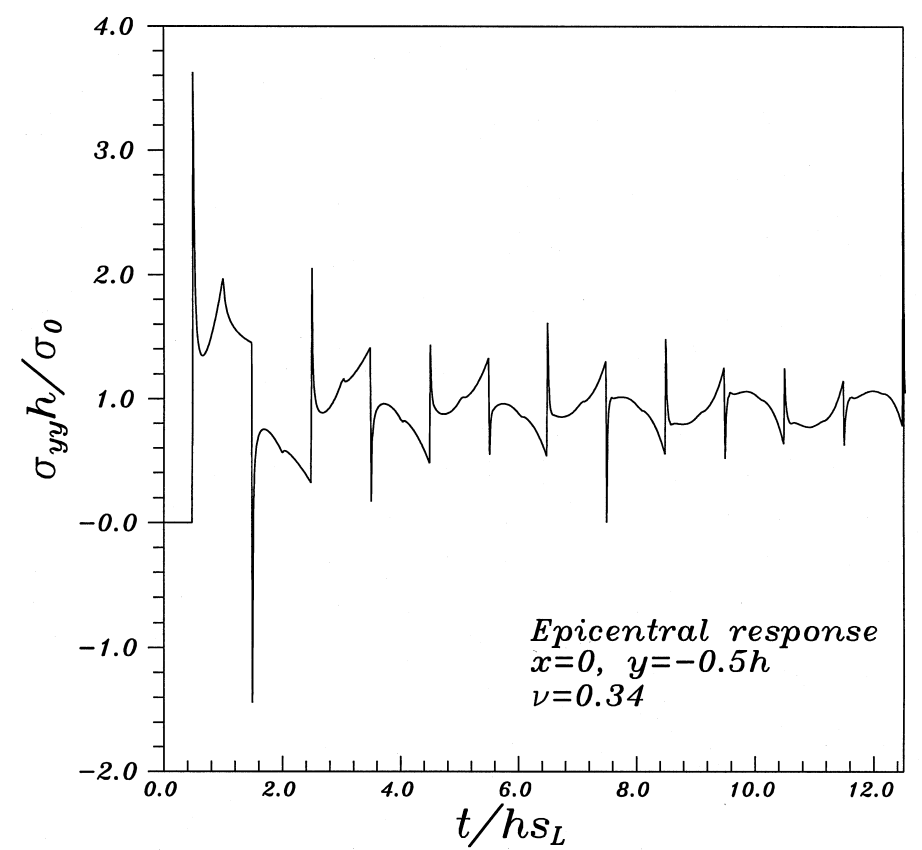

Fig. 4. The transient response of the normal stress $\sigma_{y y}$ at the epicenter.

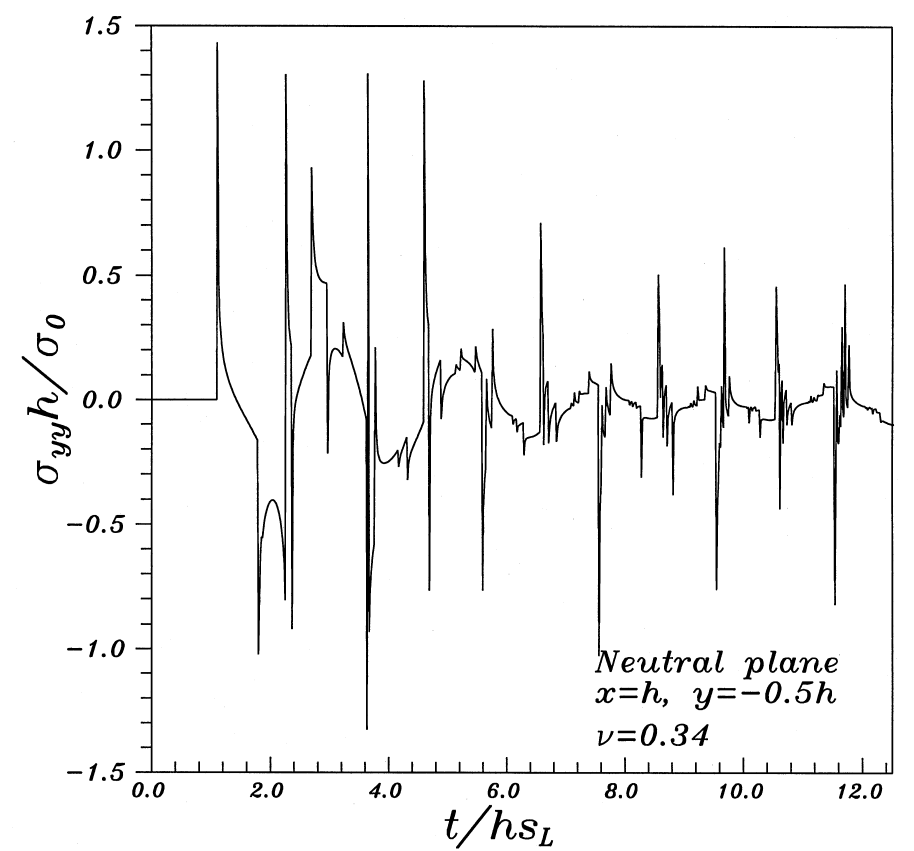

Fig. 5. The transient response of the normal stress $\sigma_{y y}$ at the position $(h,-0.5 h)$. 


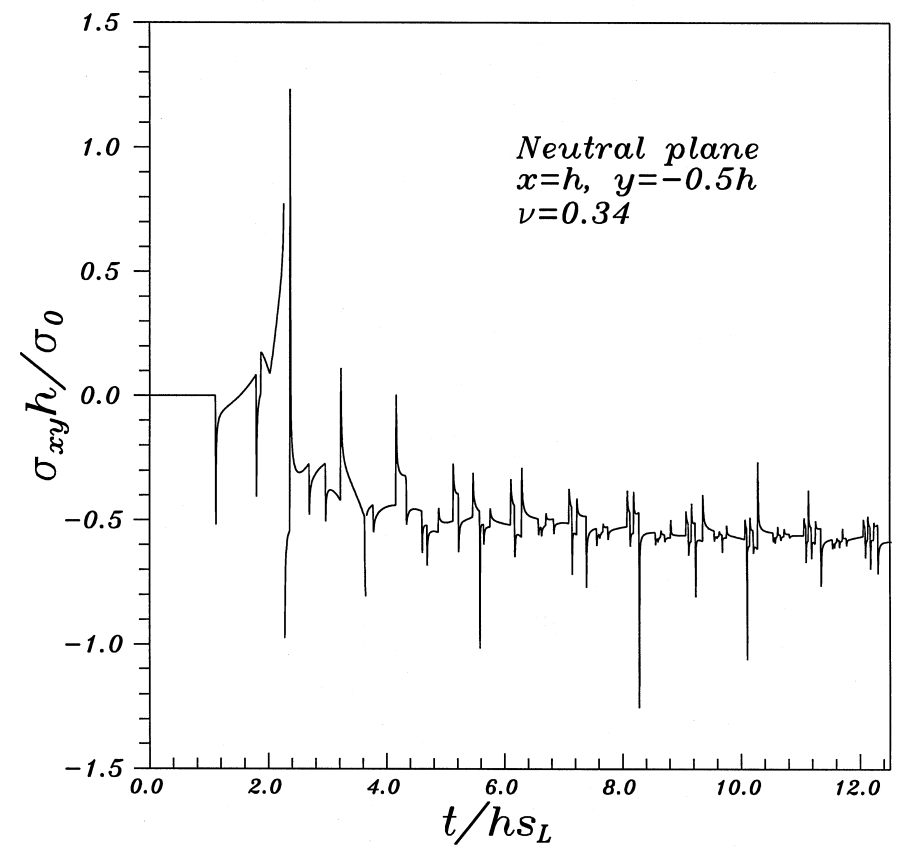

Fig. 6. The transient response of the shear stress $\sigma_{x y}$ at the position $(h,-0.5 h)$.

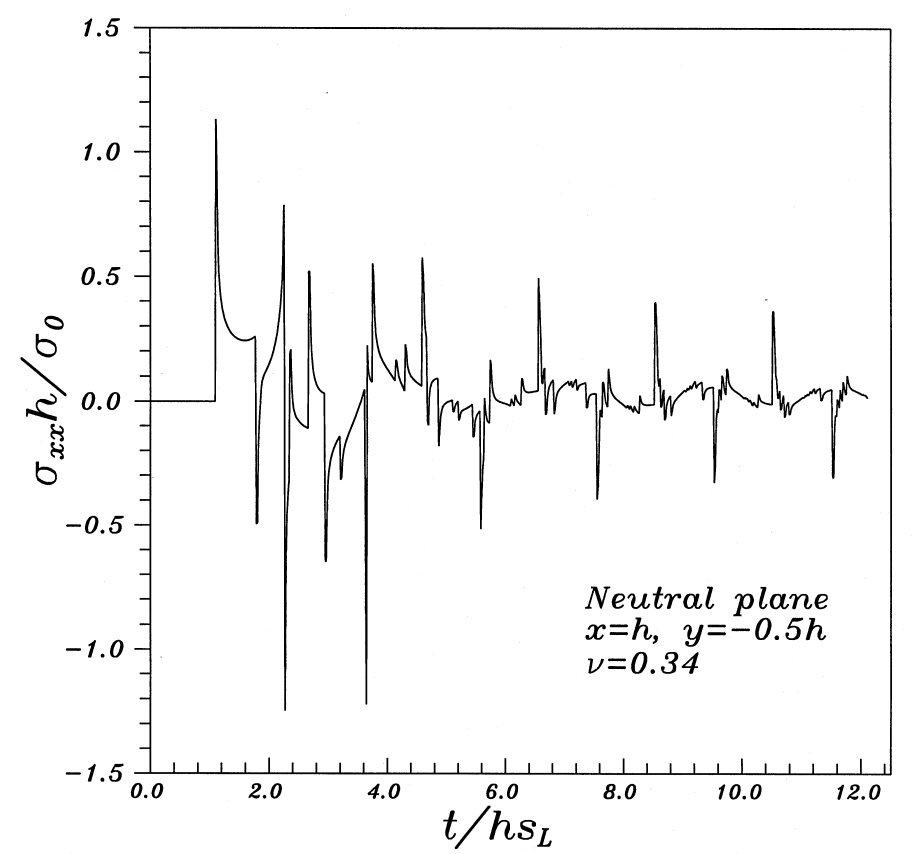

Fig. 7. The transient response of the normal stress $\sigma_{x x}$ at the position $(h,-0.5 h)$. 


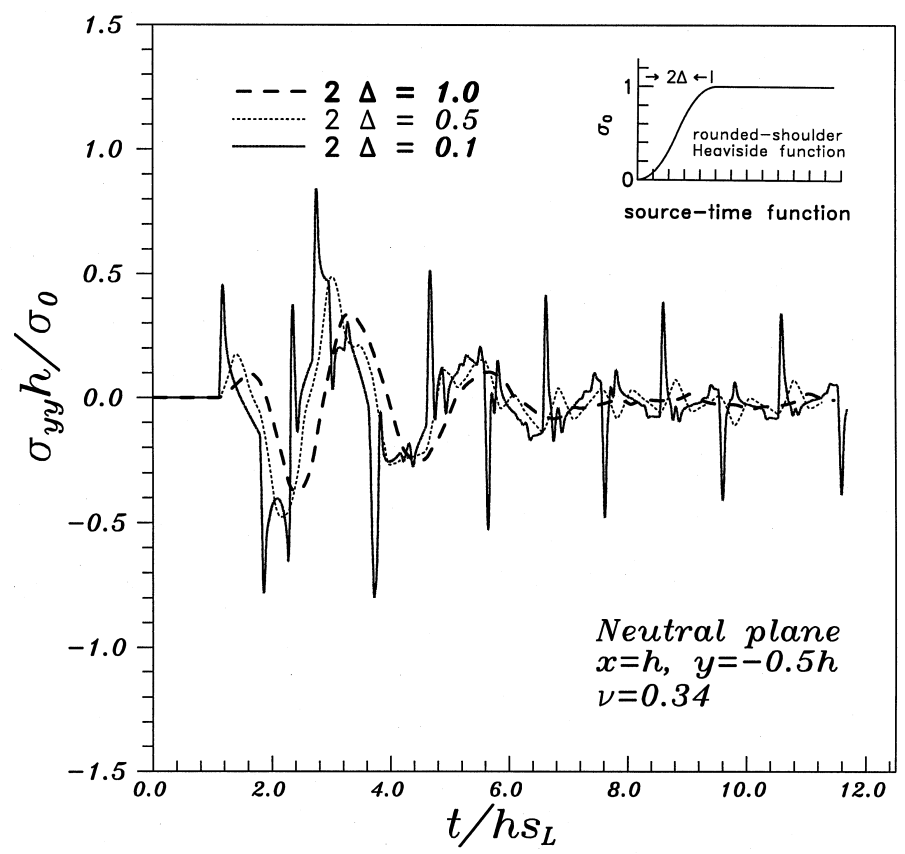

Fig. 8. The transient response of the normal stress $\sigma_{y y}$ at $(h,-0.5 h)$ for the rounded shoulder Heaviside source function.

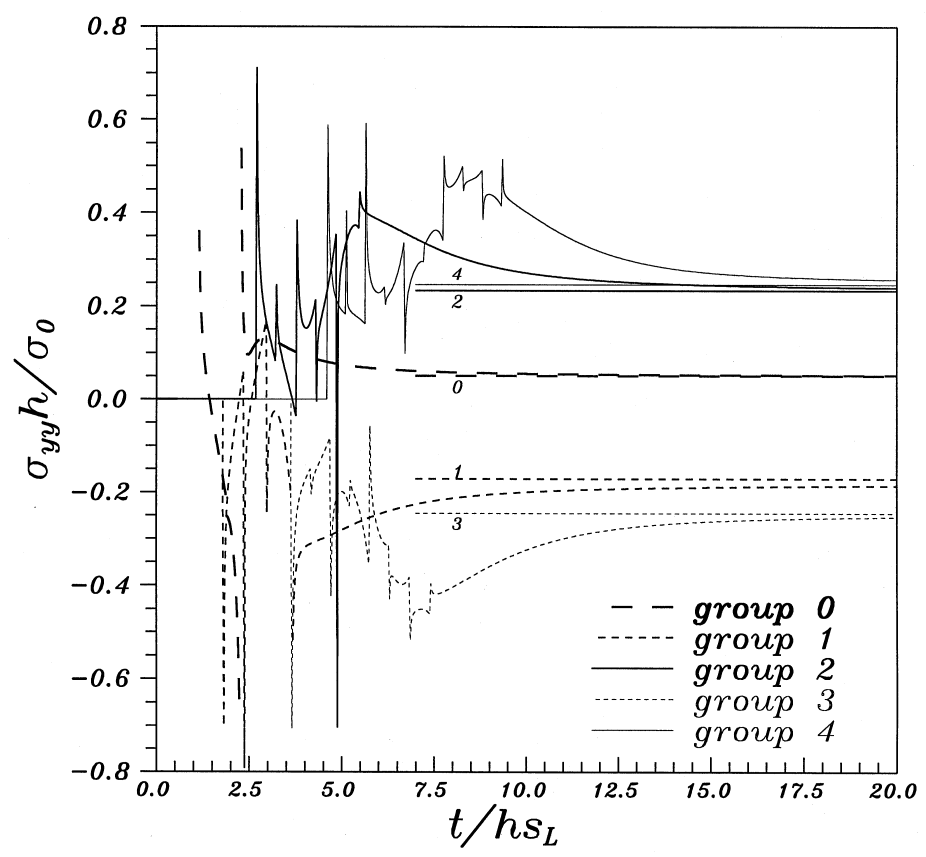

Fig. 9. The transient response of the normal stress $\sigma_{y y}$ for each group of waves at the position $(h,-0.5 h)$. 


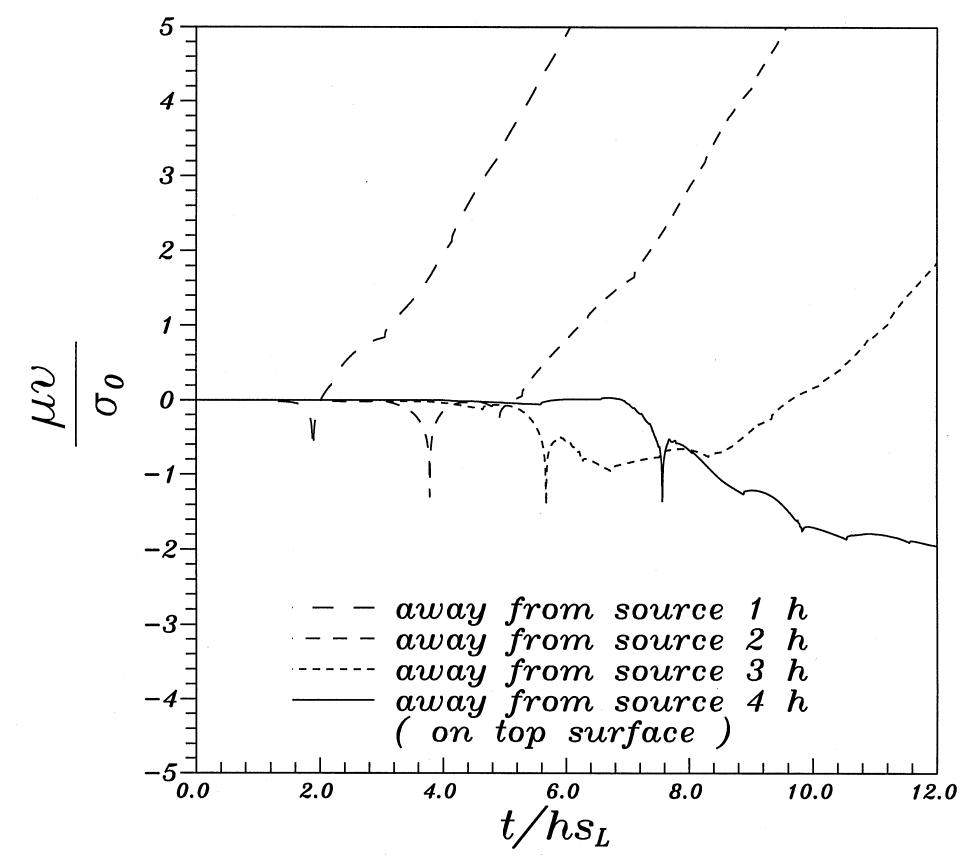

Fig. 10. The vertical displacement on the top surface at different location.

The numerical results of vertical displacements for top and lower surfaces at different positions due to applying Heaviside time dependent loading are shown in Figs 10 and 11, respectively. The strongest motion of the top surface can be easily identified as the Rayleigh surface wave arrives at the material point. However, the Rayleigh surface wave on the lower surface does not induce significant response. The discontinuity of the slope of the displacement is due to the arrival of waves which carries singularity at the wave front.

The calculated displacement on the top surface based on the analytical solution in (40b) is compared to the experimental measurement on a steel plate. The density and elastic constants of steel are

$$
\rho=7900 \mathrm{Kg} / \mathrm{m}^{3}, \quad E=207.0 \mathrm{GPa}, \quad v=0.29 .
$$

The thin plate specimen with $h=3 \mathrm{~cm}$ is supported by a holding frame as shown in Fig. 12. The dynamic loading is a step excitation concentrated force applied on the top surface of the plate. The step loading can be generated from the brittle fracture of a pencil lead and the experimental response is shown in Fig. 13. The experimental results of vertical displacements at different positions shown in Figs 14-15 are measured by an NBS conical transducer, and recorded by an oscilloscope (Lecroy 9310L). The agreements between the theoretical results and experimental measurements are excellent, which imply that the evaluation of the material constants by using the transient wave theory may be possible.

\section{Conclusion}

This paper presents a matrix expansion method for analyzing the transient waves in a strip generated by dynamic loadings. The unknown coefficients of the wave potentials in Laplace 


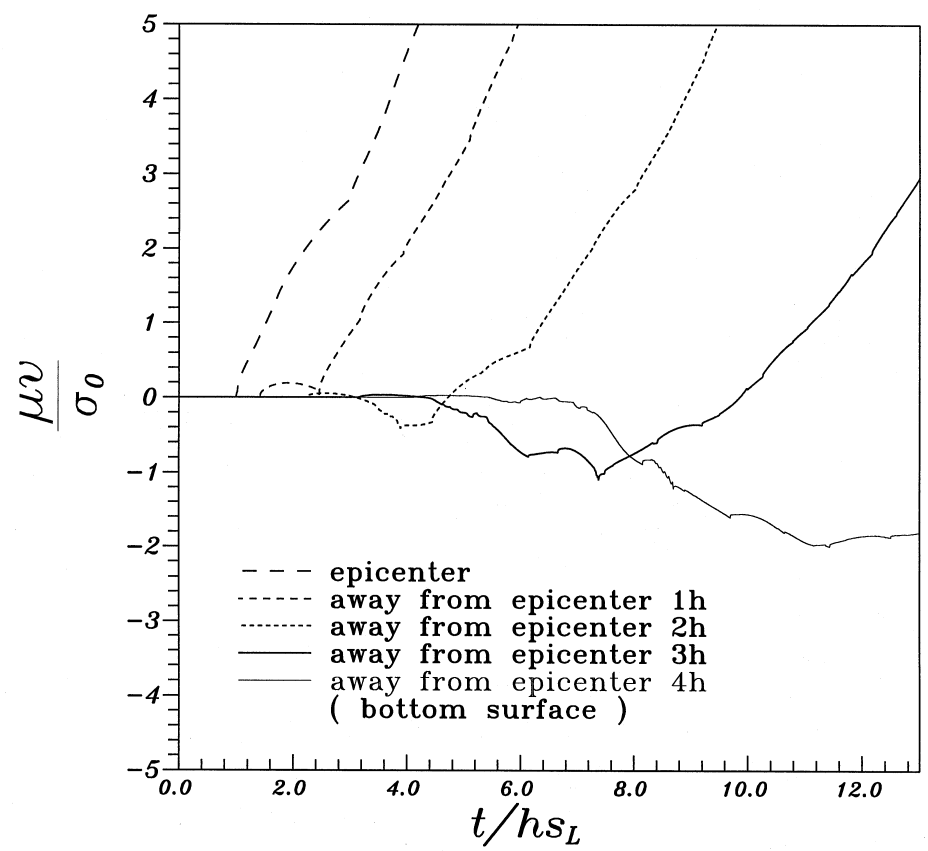

Fig. 11. The vertical displacement on the lower surface of the strip.

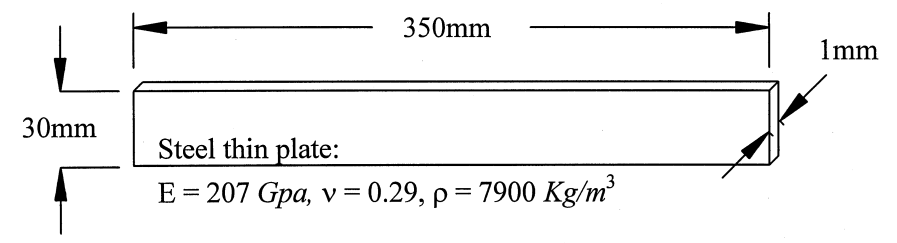

The dimension of specimen

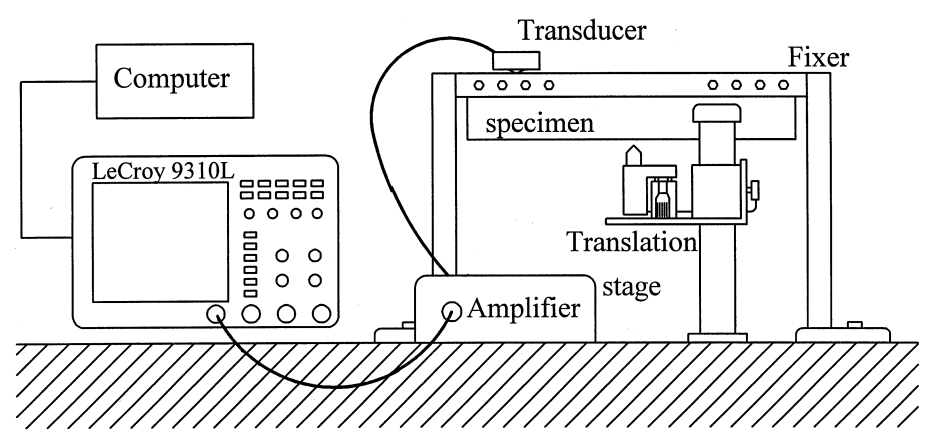

Fig. 12. The experimental setup and dimension of the specimen. 


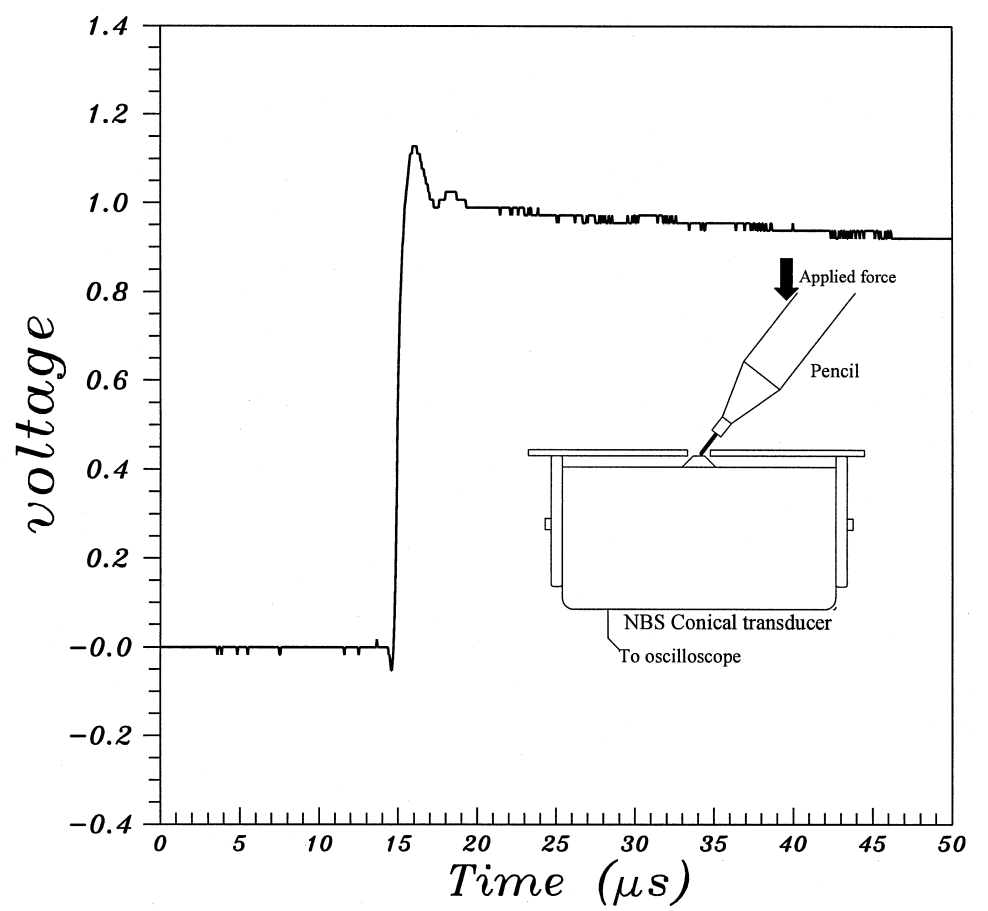

Fig. 13. The source time function produced by brittle fracture of pencil lead.

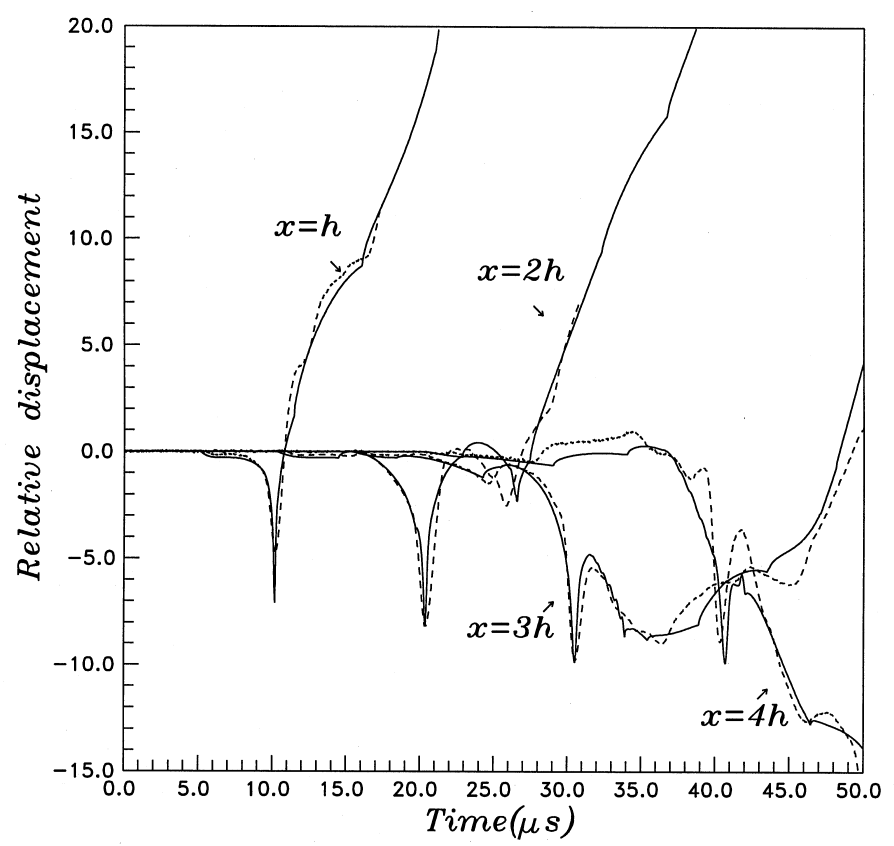

Fig. 14. The comparison between theoretical results (solid line) and experimental measurements (dashed line) of vertical displacement at the top surface. 


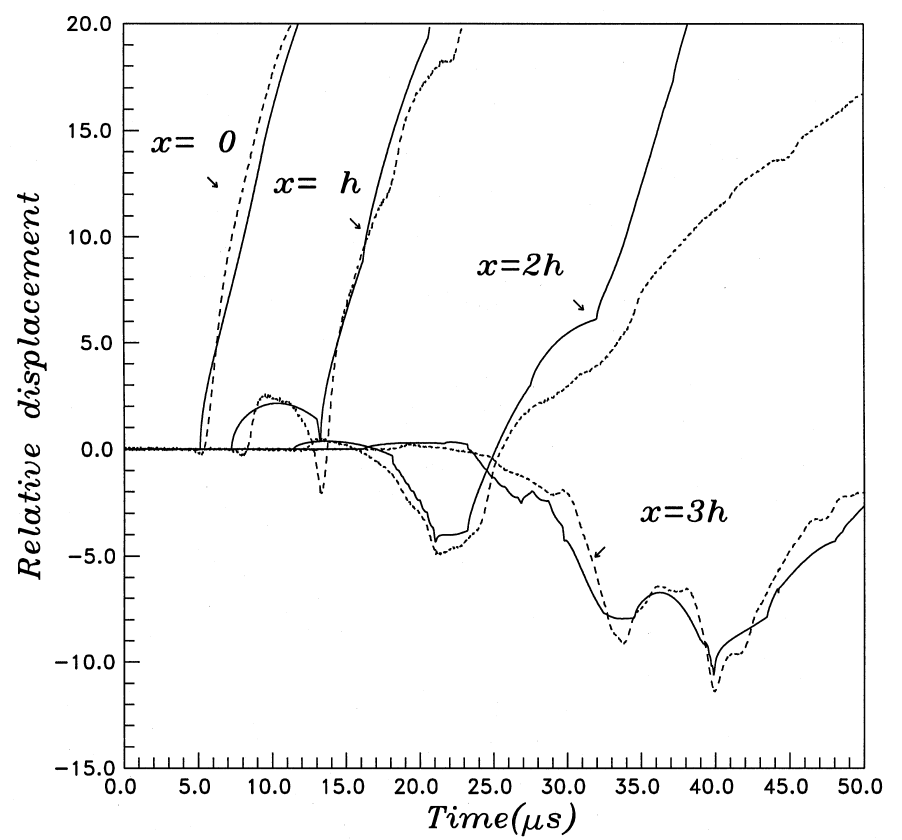

Fig. 15. The comparison between theoretical results (solid line) and experimental measurements (dashed line) of the vertical displacement at the lower surface.

transformed domain are determined from the boundary conditions at both surfaces of the strip. By rewriting the coefficient matrix $\mathbf{M}$ in a special form which consists of the diagonal, lower and upper triangular parts, the inverse transformation is accomplished without evaluating the residues by expanding $(\mathbf{I}-\mathbf{R})^{-1}$ into a power series of matrix $\mathbf{R}$. The term associated with $\mathbf{R}^{i}$ represents the group of waves that is reflected by the surfaces $i$ times. Since the matrix solution in (23) sorts the group of waves in order, only a few terms out of the infinite sum are relevant for any given time of interest. Each component of the matrix solution could be identified with the solution derived by the theory of generalized ray. The transient solution in time domain is accomplished by the application of Cagniard's method with a suitable coding of all the waves. The analytic transient results obtained in this study are exact and are expressed in a simple closed series, each term representing a physical transient wave.

In comparison with the ray expansion method proposed by Mencher (1953), Knopoff (1958) and Davids (1959), the matrix method presented in this study can construct all waves in the strip more concisely and easily. Furthermore, if the force is applied within the strip instead of the surface of the strip, the ray solution can also be obtained easily by changing the source vector $\hat{\mathbf{s}}$ accordingly without resolving the problem. The methodology provided in this study has already been successfully extended to solve more complex types of problems, which deal with the transient wave propagation in layered medium and three-dimensional configuration.

Numerical investigations of transient responses for displacements and stresses in the strip subjected to a dynamic point force is discussed in detail. Although the stress induced by each wave is diverged as time tends to infinity, the total contribution from all the waves in one group is 
converged. An experimental setup is established to measure the transient response of vertical displacement on surfaces of a strip subjected to dynamic loadings with a step time dependence. The agreements between the theoretical results and experimental measured responses are excellent, which implies the possible application of the present study to inverse evaluation of the material constants.

\section{Acknowledgement}

The financial support for the authors from the National Science Council, Republic of China, through Grant NSC86-2212-E-002-008 to National Taiwan University is gratefully acknowledged.

\section{References}

Cagniard, L., 1939. Reflexion et Refraction des Ondes Seismiques Progressives. Cauthiers-Villars, Paris; translated into English and revised by Flinn, E.A. and Dix, C.H., 1962, Reflection and Refraction of Progressive Seismic Waves. McGraw-Hill, New York.

Ceranoglu, A.N., Pao, Y.H., 1981. Propagation of elastic pulses and acoustic emission in a plate. J. App. Mech. 48, $125-147$.

Davids, N., 1959. Transient analysis of stress-wave penetration in plate. J. Appl. Mech. 26, 651-660.

De Hoop, A.T., 1961. A modification of Cagniard's method for solving seismic pulse problems. Appl. Sci. Res. B8, 349356.

Knopoff, L., 1958. Surface motion of a thick plate. J. Appl. Phys. 29, 661-670.

Lamb, H., 1904. On the propagation of tremors over the surface of an elastic solid. Philos. Trans., Royal Soc. London A203, 1-42.

Mencher, A.G., 1953. Epicentral displacement caused by elastic waves in an infinite slab. J. Appl. Phys. 24, $1240-1246$.

Miklowitz, J., 1960. Flexural stress waves in an infinite elastic plate due to a suddenly applied concentrated transverse load. J. Appl. Mech. 27, 681-689.

Miklowitz, J., 1962. Transient compressional waves in an infinite elastic plate or elastic layer overlying a rigid halfspace. J. Appl. Mech. 29, 53-60.

Norwood, F.R., 1975. Transient response of an elastic plate to loads with finite characteristic dimensions. Int. J. Solids Structures 11, 33-51.

Pao, Y.H., Gajewski, R., 1977. The generalized ray theory and transient responses of layered elastic solids. In: Mason, W.P., Thurston, R.N. (Eds.), Physical Acoustics, vol. 13. Academic Press, New York, pp. 184-266.

Santosa, F., Pao, Y.H., 1989. Transient axially asymmetric response of an elastic plate. Wave Motion 11, $271-295$.

Shmuely, M., 1975. Stress wave propagation in plates subjected to a transient line source. Int. J. Solids Structures 11, 679-691.

Spencer, T.W., 1960. The method of generalized reflection and transmission coefficients. Geophysics 25, 625-641.

Weaver, R.K., Pao, Y.H., 1982. Axisymmetric elastic waves excited by a point source in a plate. J. Appl. Mech. 49, $821-836$. 